\title{
Role of Microscopy and Microanalysis in Friction Stir Weld Development
}

\author{
M.A. Fischer * \\ * Boeing Research \& Technology, The Boeing Company, Huntington Beach, CA 92647
}

Characterization of friction stir welds, from a development as well as weld qualification and ongoing quality control measures, rely heavily on macro- and micro-structural analysis. Such characterization is not only important for an understanding of the complex plasticized state of the metal being joined, but is also a significant tool in the evaluation of defects. Micro-structural characterization, combined with non-destructive evaluation, and mechanical property determination are used to establish production weld schedules, their process sensitivities and limitations or processing windows.

Macro-structural analysis of aluminum alloy bi-metallic joints can often provide valuable clues as to the mechanics of the plasticized state and the degree of deformation occurring in the stir zone (also know as dynamic recrystallization zone (DXZ) or nugget) and Thermo-mechanically affected zone (TMAZ). The chemical etching differences between different alloys is usually sufficient to accentuate dramatic differences in the stirring action. The stirring action has also been further studied by the introduction of tracers in the joint. These tracers may consist of deposited films or sandwiched sections of ribbon or wire of a dissimilar material that can be later observed microstructurally (as with copper) or by other methods (such as radiographic inspection of tungsten tracers). Although not directly linked to mechanical properties, tracer studies may be used to understand the degree of deformation in the stir zone and TMZ given differing manufacturing variables, such as pin tool and shoulder design as well as peaking, mismatch, pin tool offset, fit-up and weld land cleaning operations. This can aid in the establishment of processing limits and potential interactions of these parameters for successful manufacturing. This is particularly true with respect to process sensitivities contributing to the development of Residual Oxide Defect (ROD). This defect, when found, can contribute to a degradation in certain mechanical properties, but is not readily detectable by traditional non-destructive evaluation methods. Therefore, proper characterization of the conditions contributing to ROD and verifiable control of such parameters should be evaluated for each application of SR-FSW that may be sensitive to this defect.

During weld development, samples found to possess indications via visual or volumetric inspection methods are often validated by metallographic analysis to confirm the type of defect as well as provide a correlation of the defect size to the response found by volumetric means. Volumetric inspection techniques are often constrained by the geometry of the hardware. Depending on the ultrasonic scan angle or orientation of radiographic source, an indication's true size can be disguised by its shape and orientation. Metallographic analysis of samples assists in understanding the likely orientations of defects within the stir zone, allowing Non-Destructive Inspection engineers to formulate the most advantageous inspection techniques to properly identify and disposition such defects when encountered. A substantial portion of weld characterization includes the volumetric inspection of a weld joint, by either radiographic or ultrasonic means to locate indications in the weld followed by destructive testing and metallographic characterization to provide correlation of defect size, location, orientation and distribution to validate and 'calibrate' the NDT techniques. 
Metallographic inspection can detect the onset of defects such as worm-hole, which can be generated during weld start-up, before steady state welding is achieved, and continue into the length of the weld if parameters are not appropriate for the joint geometry.

The most problematic of defects are those that cannot be readily discerned by nondestructive methods. Once identified, such defects must be eliminated by process control. One such example of this in aluminum alloys is ROD. The weld engineer must determine the conditions necessary for the formation of such defects using metallography and other destructive methods (tensile) and then control the manufacturing process to preclude these conditions from arising.

ROD is evaluated metallographically on cross-sectioned samples as well as on the fracture surfaces of tensile specimens. Although severe cases of ROD can be seen in polished and etched specimens, it may be necessary to evaluate samples in the un-etched condition to find ROD in less severe conditions.

Friction stir welding can be performed by either conventional (C-FSW) or self-reacting (SR-FSW) methods. ROD has been problematic for SR-FSW, but not C-FSW. In SR-FSW, oxide films present on the surface of the two members being joined may not be completely broken up or obliterated by the plasticized action of FSW. This type of defect is not sufficiently detectable by standard nondestructive techniques such as ultrasonic inspection (either conventional or phased array) or radiographic inspection. Although Phased Array Ultrasonic (PAUT) inspection may find severe cases of ROD, it may not reliably detect ROD at lower levels that still impact mechanical properties. The defect has a detrimental effect on some mechanical properties at room and cryogenic temperatures. A substantial portion of the FSW development to date has been for cryogenic fuel and oxidizer vessels (carrying liquid hydrogen and oxygen at -423 and -297 deg F respectively) for space launch vehicles. The controls and requirements governing such structures are necessarily rigorous and demanding, as are the processing and quality assurance requirements imposed during weld development and qualification.

When evaluated together; microstructural analysis, mechanical property testing and NDT results can be utilized to determine the conditions necessary for proper weld soundness and determine the appropriate cleaning, joint geometry and weld parameters for successful welding. These conditions can then be verified during weld schedule development and qualification.

TABLE 1. Weld Defect Types and Methods of Verification.

\begin{tabular}{ll}
\hline Typical FSW Defect & Non-Destructive Inspection Technique \\
\hline Cracks & Surface, Volumetric \\
Galling, Tearing, Blisters & Surface \\
Oxides, Inclusions & Surface, Volumetric \\
Voids, Worm-hole & Volumetric \\
Under Thickness & Surface, Volumetric \\
Incomplete Penetration & Surface, Volumetric \\
Incomplete Fusion & Surface, Volumetric \\
FOD (Foreign Object Damage) & Surface, Volumetric \\
ROD (Residual Oxide Defect) & None \\
\hline
\end{tabular}

